\title{
The effect of counterpoise correction and relaxation energy term to the internal rotation barriers: Application to the $\mathrm{BF}_{3} \cdots \mathrm{NH}_{3}$ and $\mathrm{C}_{2} \mathrm{H}_{4} \cdots \mathrm{SO}_{2}$ dimers
}

\author{
Pedro Salvador and Miquel Duran \\ Institute of Computational Chemistry and Department of Chemistry, University of Girona, 17071, \\ Girona, Spain
}

(Received 23 March 1999; accepted 16 June 1999)

\begin{abstract}
The relevance of the fragment relaxation energy term and the effect of the basis set superposition error on the geometry of the $\mathrm{BF}_{3} \cdots \mathrm{NH}_{3}$ and $\mathrm{C}_{2} \mathrm{H}_{4} \cdots \mathrm{SO}_{2}$ van der Waals dimers have been analyzed. Second-order Mфller-Plesset perturbation theory calculations with the $d 95(d, p)$ basis set have been used to calculate the counterpoise-corrected barrier height for the internal rotations. These barriers have been obtained by relocating the stationary points on the counterpoise-corrected potential energy surface of the processes involved. The fragment relaxation energy can have a large influence on both the intermolecular parameters and barrier height. The counterpoise correction has proved to be important for these systems. (C) 1999 American Institute of Physics. [S0021-9606(99)30634-6]
\end{abstract}

\section{INTRODUCTION}

The theoretical study of molecular interactions under the supermolecular approach with finite basis sets centered at the atomic positions originates the so-called basis set superposition error (BSSE). ${ }^{1,2}$ Within the LCAO-MO approach, each fragment can be expanded to some extent in the basis set of the partner. Thus, BSSE is the unphysical effect due to the improvement of the quantum mechanical description of the fragments within the supermolecule. While it has been recognized for a long time that this effect results in an increase of the interaction energy, ${ }^{3}$ only recently have studies of the effect of BSSE in geometrical parameters ${ }^{3,4}$ and electron density ${ }^{5}$ been addressed.

BSSE-free potential energy surfaces and interaction energies have become a target for many researchers in the last years. ${ }^{6-10}$ Under a methodological point of view, two approaches have faced up the problem. The first, aprioristic approach, constrains the supermolecule description to keep it consistent with the description of its fragments. An example of this methodology is the chemical Hamiltonian approach (CHA), introduced by Mayer, ${ }^{11}$ which eliminates BSSE-pure terms of the Hamiltonian using a mixed second quantization scheme. Several studies applying the CHA have yielded accurate results at any level of theory. ${ }^{12-15}$ Other aprioristic approximations at the Hartree-Fock level have also been recently proposed and applied to the water dimer. ${ }^{16}$

However, the most widely used method to handle BSSE has been the a posteriori counterpoise method. ${ }^{17,18}$ Boys and Bernardi and Jansen and Roos suggested that the superposition error is minimized if the same basis set is used for the description of both the supermolecule and its fragments. The literature has plenty of discussion about the validity of this approach, ${ }^{19-21}$ which stays as a matter of active controversy. This counterpoise correction scheme (CP-correction) has been shown recently ${ }^{4}$ to converge to $\mathrm{CHA}$ results for a wide range of hydrogen bonded systems, and thus it is established as a reliable procedure to study intermolecular complexes.
In the literature, the counterpoise correction for intermolecular complexes has usually been applied to estimate the BSSE-free interaction energy. In most cases it has been applied at the optimized supermolecular geometry and neglecting the relaxation of the fragments geometry with respect to the monomers situation. One can argue that this restriction is acceptable if the relaxation contribution is negligible or smaller than the desired accuracy. On the other hand, the $\mathrm{CP}$-correction depends upon the partitioning into fragments of the system. Regarding intermolecular complexes, chemical intuition helps to define as many fragments as molecules the complex exhibits. In this way, one can obtain the supermolecule structure and the energy to be consistent with the fragments defined previously. However, one can choose a more physical partitioning. One can consider that the supermolecule is made up of a set of atoms and therefore the BSSE can be seen as an intramolecular property, independent of any chemical partitioning. The last option is mandatory in case of most chemical reactions, where the counterpoise method is rather inappropriate. ${ }^{23}$ However, one of the chemical processes where fragments are defined continuously constant along the reaction coordinate is the internal rotation in weakly bonded systems. In the last years, Rayón et al. have been questioning the validity of the counterpoise correction in the evaluation of energy barriers to internal rotation including the fragment relaxation term..$^{22-24}$

The concept of the BSSE fragment relaxation term is misleading. The expression for the BSSE-correction within the counterpoise approach is defined disregarding the fragments of the system being allowed or forbidden to relax their own geometry. One cannot split the BSSE-correction term into a "relaxation term" and an "intermolecular term." When the parameters are frozen, the BSSE amount depends only on the intermolecular distances and angles, but the value of the BSSE at a given geometry depends conceptually on all the parameters involved in the calculation. Nevertheless, experience shows that the intramolecular parameters are 
not very modified when correcting for the BSSE.

The papers by Rayón et al. have assumed that there is no BSSE at all in the evaluation of energy barriers to internal rotation processes. For a system $S$, the value of the energy barrier is easily calculated as

$$
E_{b}(S)=E_{\mathrm{TS}}(S)-E_{\min }(S),
$$

with $E_{\min }$ and $E_{\mathrm{TS}}$ being the energy of the minima and the connecting transition state structure involved in the process, respectively. Consistent results can be obtained provided that the system is properly described. However, both $E^{\mathrm{TS}}$ and $E^{\min }$ and hence $E_{b}$ are BSSE-contaminated, i.e., if the system $S$ is made up of two subsystems $A$ and $B$. This is the case for weakly bonded complexes. Thus, the energy barrier should be calculated as

$$
\begin{aligned}
E_{b}^{\mathrm{CP}}(S) & =E_{\mathrm{TS}}^{\mathrm{CP}}(S)-E_{\min }^{\mathrm{CP}}(S) \\
& =E_{\mathrm{TS}}(S)-E_{\min }(S)+\delta_{\mathrm{TS}}^{\mathrm{CP}}(S)-\delta_{\min }^{\mathrm{CP}}(S) .
\end{aligned}
$$

The assumption of zero BSSE is only valid if the last two terms vanish, i.e., if BSSE were independent of the geometry, which is not at all the case. Therefore, it can be important to relocate the structures in the CP-corrected PES. Note that both $E_{\mathrm{TS}}^{\mathrm{CP}}(S)$ and $E_{\mathrm{min}}^{\mathrm{CP}}(S)$ can be obtained with the counterpoise receipt regardless of the fragment relaxation being included or excluded.

To gain a deeper insight on this point we have performed both uncorrected and $\mathrm{CP}$-corrected geometry optimizations for two weakly bonded subsystems, $\mathrm{BF}_{3} \cdots \mathrm{NH}_{3}$ and $\mathrm{C}_{2} \mathrm{H}_{4} \cdots \mathrm{SO}_{2}$ and compared the values obtained for the energy barriers using Eqs. (1) and (2) with those obtained by Rayón et al. ${ }^{24}$ We have analyzed also the differences between the so-called single point counterpoise correction, i.e., the energy correction at the uncorrected PES (s.p. CP-correction), and the $\mathrm{CP}$-corrected optimization. Finally, we decided to study the effect of the fragment relaxation to both the energy and the geometry for these systems. Therefore, we have reoptimized the geometry with the intramolecular parameters frozen at the optimum value of the free monomers.

To better understand the implications of the BSSE and the fragment relaxation, in the following section we will present first compact formulas for the influence of the counterpoise correction on the geometry of a chemical system. Then, formulas will be given for the analysis of the fragment relaxation, with particular focus on the case of an internal rotation process.

In the second part of the paper we will describe the computational procedure, whereas the third part will deal with the discussion of the results obtained and their analysis.

\section{A. Counterpoise correction to geometry}

Let us consider a supermolecule $A B$ made up of two interacting subsystems $A$ and $B$. The interaction energy can be expressed as

$$
\Delta E(A B)=E_{A B}^{A B}(A B)-E_{A}^{A}(A)-E_{B}^{B}(B) .
$$

We define $E_{Y}^{Z}(X)$ as the energy of subsystem $X$ at geometry $Y$ with basis set $Z$. The interaction energy can be split in the following way:

$$
\Delta E(A B)=\Delta E_{\mathrm{el}}(A B)+\Delta E_{\mathrm{rel}}(A, B) .
$$

The first term represents the electronic contribution, which depends only on the supermolecule geometrical parameters, $\{A B\}$,

$$
\Delta E_{\mathrm{el}}(A B)=E_{A B}^{A B}(A B)-E_{A B}^{A}(A)-E_{A B}^{B}(B),
$$

whereas the second term, positive definite, represents the relaxation contribution, ${ }^{25}$ which compensates for the geometry distortion of the subsystems in the supermolecule, $E_{A B}^{A}(A)$ and $E_{A B}^{B}(B)$, with regard to the isolated optimum geometry, $E_{A}^{A}(A)$ and $E_{B}^{B}(B)$,

$$
\Delta E_{\mathrm{rel}}(A, B)=E_{A B}^{A}(A)-E_{A}^{A}(A)+E_{A B}^{B}(B)-E_{B}^{B}(B) .
$$

Note that $\Delta E_{\text {rel }}(A, B)$ depends on both the supermolecule and subsystem parameters, $\{A B, A, B\}$.

According to the counterpoise idea, since the same basis set is used in the relaxation term for each subsystem, only the electronic contribution term brings about the BSSE. Thus, the counterpoise-corrected interaction energy should be written as

$$
\begin{aligned}
\Delta E^{\mathrm{CP}}(A B)= & {\left[E_{A B}^{A B}(A B)-E_{A B}^{A B}(A)-E_{A B}^{A B}(B)\right] } \\
& +\left[E_{A B}^{A}(A)+E_{A B}^{B}(B)-E_{A}^{A}(A)-E_{B}^{B}(B)\right] \\
= & {\left[E_{A B}^{A B}(A B)-E_{A}^{A}(A)-E_{B}^{B}(B)\right] } \\
& +\left[E_{A B}^{A}(A)+E_{A B}^{B}(B)-E_{A B}^{A B}(A)-E_{A B}^{A B}(B)\right] \\
= & \Delta E(A B)+\delta_{A B}^{\mathrm{BSSE}},
\end{aligned}
$$

where the CP-correction expressed as $\delta_{A B}^{\mathrm{BSSE}}$ tends to zero as the basis sets of the subsystems approach completeness and obviously depends on the supermolecular structural parameters, as shown by the subscript $A B$. Thus, BSSE is not an additive term to the interaction energy. Indeed, it is strongly geometry-dependent ${ }^{4}$ and can modify meaningfully the uncorrected, BSSE-contaminated geometrical parameters and vibrational properties. ${ }^{3,4}$

Any stationary point of the uncorrected supermolecule potential energy surface determines a stationary point of the interaction energy surface, because there is no variation in the isolated subsystems. Differentiating Eq. (7),

$$
\begin{aligned}
\frac{\partial\left(\Delta E^{\mathrm{CP}}(A B)\right)}{\partial R_{i}}= & \frac{\partial(\Delta E(A B))}{\partial R_{i}}+\frac{\partial\left(\delta_{A B}^{\mathrm{BSSE}}\right)}{\partial R_{i}} \\
= & \frac{\partial\left(E_{A B}^{A B}(A B)\right)}{\partial R_{i}}+\frac{\partial\left(E_{A B}^{A}(A)\right)}{\partial R_{i}} \\
& +\frac{\partial\left(E_{A B}^{B}(B)\right)}{\partial R_{i}}-\frac{\partial\left(E_{A B}^{A B}(A)\right)}{\partial R_{i}} \\
& -\frac{\partial\left(E_{A B}^{A B}(B)\right)}{\partial R_{i}} \quad \forall R_{i} \in\{A B\},
\end{aligned}
$$




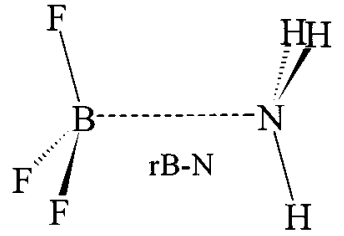

Minimum

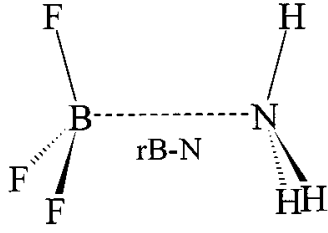

Transition State
FIG. 1. Structures involved in the internal rotation of the $\mathrm{BF}_{3} \cdots \mathrm{NH}_{3}$ system. Intermolecular distance is indicated.

it can be seen that the uncorrected supermolecular geometry is only valid under the assumption that the BSSE defined as $\delta_{A B}^{\mathrm{BSSE}}$ is stationary at the current nuclear arrangement,

$$
\frac{\partial\left(\delta_{A B}^{\mathrm{BSSE}}\right)}{\partial R_{i}}=0 \quad \forall R_{i} \in\{A B\} .
$$

In order to obtain corrected interaction energies, one has to deal with a corrected supermolecular potential energy surface. The counterpoise corrected PES for the supermolecule can be defined as follows:

$$
\begin{aligned}
E^{\mathrm{CP}}(A B)= & E_{A B}^{A B}(A B)+\delta_{A B}^{\mathrm{BSSE}}=E_{A B}^{A B}(A B) \\
& +\left[E_{A B}^{A}(A)+E_{A B}^{B}(B)-E_{A B}^{A B}(A)-E_{A B}^{A B}(B)\right] .
\end{aligned}
$$

The equation above represents another point of view of the $\mathrm{CP}$-correction. In our opinion, it should be more generally assigned to the supermolecule description, rather than to the interaction energy. Some authors ${ }^{22}$ have argued that BSSE is a pure interaction energy term; however, BSSE exists even thought we are not interested in interaction energy. In fact, $E_{A B}(A B)$ and $\Delta E_{A B}(A B)$ differ by a BSSE-free constant term which depends only on the system and the definition of the fragments. Therefore it seems quite coherent to assign the BSSE correction to $E_{A B}(A B)$.

Equation (8) can be easily generalized to the $N$ th-order energy derivatives. Second and third derivatives to be used for both harmonic and anharmonic vibrational analysis can be expressed as linear combinations of the contributions of each term, as shown by Simon et $a l^{3}$ Any property defined as a derivative of the energy can be corrected for the BSSE. Unlike CHA methods, there is no definition for a $\mathrm{CP}$ corrected electronic density for the supermolecule.

\section{B. The role of the fragment relaxation}

To estimate the BSSE-free interaction energy neglecting the relaxation of the fragments geometry one can rewrite Eq. (7) as

$$
\Delta E_{\text {no-rel }}^{\mathrm{CP}}(A B)=E_{A B}^{A B}(A B)-E_{A B}^{A B}(A)-E_{A B}^{A B}(B) .
$$

As mention above, this expression can be acceptable if the relaxation contribution is negligible or smaller than the desired accuracy. This equation usually refers to the relaxation of the fragments at the uncorrected supermolecule geometry, which is indeed different when corrected for BSSE by the counterpoise method. One must assume that the energetic relaxation contribution at both the corrected and uncorrected supermolecule geometry should be of the same size. Recent calculations have shown that intermolecular parameters were the only ones modified meaningfully when correcting through counterpoise method. ${ }^{3,4}$ Thus, the contribution of the relaxation term on the interaction energy is almost constant. Derivative of Eq. (6) shows

$$
\begin{aligned}
\frac{\partial\left(\Delta E_{\mathrm{rel}}(A B)\right)}{\partial R_{i}} & =\frac{\partial\left(E_{A B}^{A}(A)+E_{A B}^{B}(B)+E_{A}^{A}(A)-E_{B}^{B}(B)\right)}{\partial R_{i}} \\
& =\frac{\partial\left(E_{A B}^{A}(A)\right)}{\partial R_{i}}+\frac{\partial\left(E_{A B}^{B}(B)\right)}{\partial R_{i}} \quad \forall R_{i} \in\{A, B\},
\end{aligned}
$$

where $E_{A}^{A}(A)$ and $E_{B}^{B}(B)$ vanish by definition. Derivatives involving intermolecular parameters also vanish because both fragment contributions are calculated with their own basis set. Furthermore, differentiating the interaction energy expression one obtains

TABLE I. Geometrical parameters, electronic energies, and relaxation contribution for the minimum energy structure of $\mathrm{BF}_{3} \cdots \mathrm{NH}_{3}$ complex calculated at MP2, s.p.CP-MP2, and CP-MP2 levels of theory (see Fig. 1). Number of basis functions is shown. The second half collects the values obtained in the calculations where the intramolecular parameters were frozen to the values they have in the free monomers. Relaxation energy is calculated using Eq. (6)

\begin{tabular}{lcccccccc}
\hline \hline \multicolumn{1}{c}{ Method } & $\begin{array}{c}\text { Basis } \\
\text { functions }\end{array}$ & $\begin{array}{c}\text { Electronic energy } \\
\text { (hartrees) }\end{array}$ & $\begin{array}{c}r \mathrm{~B}-\mathrm{N} \\
(\AA)\end{array}$ & $\begin{array}{c}r \mathrm{~B}-\mathrm{F} \\
(\AA)\end{array}$ & $\begin{array}{c}a \mathrm{FBN} \\
(\mathrm{deg})\end{array}$ & $\begin{array}{c}r \mathrm{~N}-\mathrm{H} \\
(\AA)\end{array}$ & $\begin{array}{c}a \mathrm{HNB} \\
(\mathrm{deg})\end{array}$ & $\begin{array}{c}\text { Relaxation } \\
\text { energy } \\
\left(\mathrm{cm}^{-1}\right)\end{array}$ \\
\hline $\begin{array}{l}\text { MP2/d95(d,p) } \\
\text { s.p.CP-corrected }\end{array}$ & 95 & -380.2945412 & 1.671 & 1.377 & 104.1 & 1.019 & 110.5 & 59 \\
CP-MP2/d95(d,p) & 95 & -380.2820718 & 1.725 & 1.375 & 103.5 & 1.018 & 110.4 & 55 \\
$\begin{array}{l}\text { MP2/d95 }(d, p) \\
\text { s.p.CP-corrected }\end{array}$ & 95 & -380.2682417 & 2.383 & $1.321^{\mathrm{a}}$ & $90.0^{\mathrm{a}}$ & $1.015^{\mathrm{a}}$ & $112.2^{\mathrm{a}}$ & $0^{\mathrm{b}}$ \\
CP-MP2/d95(d,p) & 95 & -380.2639227 & 2.519 & $1.321^{\mathrm{a}}$ & $90.0^{\mathrm{a}}$ & $1.015^{\mathrm{a}}$ & $112.2^{\mathrm{a}}$ & $0^{\mathrm{b}}$ \\
\hline \hline
\end{tabular}

${ }^{\mathrm{a}}$ Optimized parameters for $\mathrm{BF}_{3}$ and $\mathrm{NH}_{3}$ systems.

bero by definition. 
TABLE II. Geometrical parameters, electronic energies, and relaxation contribution for the transition state structure of the $\mathrm{BF}_{3} \cdots \mathrm{NH}_{3}$ complex calculated at MP2, s.p.CP-MP2, and CP-MP2 levels of theory (see Fig. 1). Number of basis functions is shown. The second half collects the values obtained in the calculations where the intramolecular parameters were frozen to the values they have in the free monomers. Relaxation energy is calculated using Eq. (6).

\begin{tabular}{lcccccccc}
\hline \hline \multicolumn{1}{c}{ Method } & $\begin{array}{c}\text { Basis } \\
\text { functions }\end{array}$ & $\begin{array}{c}\text { Electronic energy } \\
\text { (hartrees) }\end{array}$ & $\begin{array}{c}r \mathrm{~B}-\mathrm{N} \\
(\AA)\end{array}$ & $\begin{array}{c}r \mathrm{~B}-\mathrm{F} \\
(\AA)\end{array}$ & $\begin{array}{c}a \mathrm{FBN} \\
(\mathrm{deg})\end{array}$ & $\begin{array}{c}r \mathrm{~N}-\mathrm{H} \\
(\AA)\end{array}$ & $\begin{array}{c}a \mathrm{HNB} \\
(\mathrm{deg})\end{array}$ & $\begin{array}{c}\text { Relaxation } \\
\text { energy } \\
\left(\mathrm{cm}^{-1}\right)\end{array}$ \\
\hline $\begin{array}{l}\text { MP2/d95(d,p) } \\
\text { s.p.CP-corrected }\end{array}$ & 95 & -380.2924572 & 1.687 & 1.377 & 104.2 & 1.018 & 110.4 & 58 \\
CP-MP2/d95(d,p) & 95 & -380.2802673 & 1.747 & 1.374 & 103.5 & 1.018 & 110.4 & 54 \\
MP2/d95(d,p) & 95 & -380.2677108 & 2.411 & $1.321^{\mathrm{a}}$ & $90.0^{\mathrm{a}}$ & $1.015^{\mathrm{a}}$ & $112.2^{\mathrm{a}}$ & $0^{\mathrm{b}}$ \\
s.p.CP-corrected & & -380.2632994 & & & & & & \\
CP-MP2/d95(d,p) & 95 & -380.2637303 & 2.533 & $1.321^{\mathrm{a}}$ & $90.0^{\mathrm{a}}$ & $1.015^{\mathrm{a}}$ & $112.2^{\mathrm{a}}$ & $0^{\mathrm{b}}$ \\
\hline \hline
\end{tabular}

${ }^{a}$ Optimized parameters for $\mathrm{BF}_{3}$ and $\mathrm{NH}_{3}$ systems.

bero by definition.

$$
\begin{aligned}
\frac{\partial\left(\Delta E_{\mathrm{no}-\mathrm{rel}}^{\mathrm{CP}}(A B)\right)}{\partial R_{i}}= & \frac{\partial\left(E_{A B}^{A B}(A B)\right)}{\partial R_{i}}-\frac{\partial\left(E_{A B}^{A B}(A)\right)}{\partial R_{i}} \\
& -\frac{\partial\left(E_{A B}^{A B}(B)\right)}{\partial R_{i}} \quad \forall R_{i} \in\left\{A B^{\prime}\right\},
\end{aligned}
$$

where $\left\{A B^{\prime}\right\}$ represents merely the intermolecular parameters, i.e., the intermolecular distance and angular variables. The relaxation term does not contribute directly to the CPcorrected intermolecular parameters. The values can differ from those obtained using Eq. (8) due to the presence of the last two terms. Equation (10) is analogous to Eq. (7); in the complete basis set limit Eq. (10) does not converge to Eq. (7), so that the neglect of relaxation terms can be important even in the case of large basis sets.

\section{COMPUTATIONAL DETAILS}

$A b$ initio calculations were carried out using the GAUSSIAN94 package. ${ }^{26} \mathrm{MP} 2$ (Ref. 27) geometry optimizations for $\mathrm{BF}_{3} \cdots \mathrm{NH}_{3}$ and $\mathrm{C}_{2} \mathrm{H}_{4} \cdots \mathrm{SO}_{2}$ van der Waals complexes were carried out with the $d 95(d, p)$ (Ref. 28) basis set. The frozen-core approximation was also used throughout. CPcorrected geometry optimizations were performed as described by Simon et al. ${ }^{3}$ The convergence criterion was set to $10^{-5}$ a.u. in the RMS gradient. A Mixed BFGS-DIIS (Refs. $29,30)$ method was used for the location of the stationary structures on the CP-corrected PES.

\section{RESULTS AND DISCUSSION}

Figure 1 depicts the structures involved in the internal rotation along the $C_{3}$ axis for the $\mathrm{BF}_{3} \cdots \mathrm{NH}_{3}$ complex. Tables I and II collect the total energies and relevant geometric parameters for the minimum and transition state, respectively. In both cases, uncorrected and CP-corrected optimizations have been carried out. Uncorrected numbers are in perfect agreement with those obtained by Rayón et al. ${ }^{24}$ As expected, the CP-corrected intermolecular distance $(r \mathrm{~B}-\mathrm{N})$ is larger than the uncorrected one. Differences of the order of $0.05 \AA$ are observed for both the eclipsed and alternated geometry. However, the effect of BSSE on the intramolecular distances and angles is very small. A maximum difference of $0.7^{\circ}$ and $0.003 \AA$ is found for the $a \mathrm{BFN}$ angle and $r \mathrm{~B}-\mathrm{F}$ distance, respectively in the TS structure. s.p.CP-correction overcorrects the BSSE by about $0.2-0.3$ $\mathrm{kcal} / \mathrm{mol}$.

One of the main goals of this paper is the analysis of the relaxation term in both the uncorrected and CP-corrected energy and geometry. In Tables I and II we also present the result of the optimization of the system with intramolecular variables fixed to the values they exhibit in free fragments (e.g., $\mathrm{BF}_{3}$ being planar). Thus, the intermolecular interaction is studied also, by keeping fixed intramolecular parameters, to assess the importance of fragment geometry relaxation on the intermolecular geometries and energetics.

The relaxation energy is BSSE-independent in this system. The energy penalty falls in the range of $54-59 \mathrm{~cm}^{-1}$ at both the minimum and TS geometry, independently of the $\mathrm{CP}$-correction being applied. However, the effect on the $\mathrm{B}-\mathrm{N}$ distance is dramatic. For both structures, $r \mathrm{~B}-\mathrm{N}$ is lengthened by about $0.7-0.8 \AA$ when the fragments are not allowed to relax. The CP-correction increases this number even more. Thus, the BSSE effect on the geometry is more important without the relaxation term, differences of $\approx 0.13$ being observed here.

Table III shows the calculated values for the barrier height to internal rotation. It can be seen that it is not strongly affected by the CP-correction. That means that BSSE-contamination is similar for both the minimum and the TS. Using Eq. (1) we obtained 457 and $408 \mathrm{~cm}^{-1}$ for the uncorrected and s.p.CP-corrected energies, respectively, in good agreement with Rayón et al. ${ }^{24}$ Use of the CP-corrected

TABLE III. Electronic energies $\left(\mathrm{cm}^{-1}\right)$ for the internal rotation barrier in the $\mathrm{BF}_{3} \cdots \mathrm{NH}_{3}$ complex. In parentheses the values are obtained neglecting the relaxation term.

\begin{tabular}{llr}
\hline \hline \multicolumn{1}{c}{ Method } & \multicolumn{2}{c}{ Rotational barrier $\left(\mathrm{cm}^{-1}\right)$} \\
\hline MP2/d95( $d, p)$ & 457 & $(117)$ \\
s.p.CP-corrected & 408 & $(33)$ \\
CP-MP2/d95 $(d, p)$ & 396 & $(42)$ \\
\hline \hline
\end{tabular}


TABLE IV. Geometrical parameters, electronic energies, and relaxation contribution for the minimum energy structure of the $\mathrm{C}_{2} \mathrm{H}_{4} \cdots \mathrm{SO}_{2}$ complex calculated at MP2, s.p.CP-MP2, and CP-MP2 levels of theory (see Fig. 2). Number of basis functions is shown. The second half collects the values obtained in the calculations where the intramolecular parameters were frozen to the values they have in the free monomers. Relaxation energy is calculated using Eq. (6).

\begin{tabular}{lcccccccc}
\hline \hline \multicolumn{1}{c}{ Method } & $\begin{array}{c}\text { Basis } \\
\text { functions }\end{array}$ & $\begin{array}{c}\text { Electronic energy } \\
(\text { hartrees })\end{array}$ & $\begin{array}{c}R \\
(\AA)\end{array}$ & $\begin{array}{c}\alpha \\
(\mathrm{deg})\end{array}$ & $\begin{array}{c}\beta \\
(\mathrm{deg})\end{array}$ & $\begin{array}{c}a \mathrm{SOO} \\
(\mathrm{deg})\end{array}$ & $\begin{array}{c}a \mathrm{HCH} \\
(\mathrm{deg})\end{array}$ & $\begin{array}{c}\text { Relaxation } \\
\text { energy } \\
\left(\mathrm{cm}^{-1}\right)\end{array}$ \\
\hline $\begin{array}{l}\text { MP2/d95(d,p) } \\
\text { s.p.CP-corrected }\end{array}$ & 108 & -626.0441776 & 3.465 & 85.0 & 19.7 & 108.6 & 121.3 & 1 \\
CP-MP2/d95(d,p) & 108 & -626.0386655 & & & & & & \\
$\begin{array}{l}\text { MP2/d95(d,p) } \\
\text { s.p.CP-corrected }\end{array}$ & 108 & -626.0438002 & 3.474 & 84.0 & 20.1 & $108.9^{\mathrm{a}}$ & $120.0^{\mathrm{a}}$ & $0^{\mathrm{b}}$ \\
CP-MP2/d95 $(d, p)$ & 108 & -626.0386331 & 3.687 & 91.0 & 15.0 & $108.9^{\mathrm{a}}$ & $120.0^{\mathrm{a}}$ & $0^{\mathrm{b}}$ \\
\hline \hline
\end{tabular}

${ }^{a}$ Optimized parameters for $\mathrm{C}_{2} \mathrm{H}_{4}$ and $\mathrm{SO}_{2}$ isolated systems.

bero by definition.

receipt [Eq. (2)] decreases that value to $396 \mathrm{~cm}^{-1}$. The effect of the relaxation term is again very important. The calculated values are much smaller when the fragments are undistorted than in the full optimization. The uncorrected value decreases to $117 \mathrm{~cm}^{-1}$, whereas for s.p.CP- and CP-corrected rotations the barrier height is only 33 and $42 \mathrm{~cm}^{-1}$, respectively. Note that whereas the relaxation energy at both the eclipsed and alternated geometries is $\approx 58 \mathrm{~cm}^{-1}$, the difference in the barrier height turns out to be $\approx 350 \mathrm{~cm}^{-1}$. The same happens for the CP-corrected calculations. These results show clearly the importance of the effect of the relaxation term on the geometry.

Tables IV and V collect the geometrical parameters obtained for the $\mathrm{C}_{2} \mathrm{H}_{4} \cdots \mathrm{SO}_{2}$ system at both the minimum and transition state structures (see Fig. 2). Only selected intramolecular parameters are shown. Regarding the BSSE effect, a trend similar to that found for the first system is observed. The s.p.CP-correction overestimates BSSE in all the cases. $\mathrm{CP}$-corrected intermolecular distance $R$ is $\approx 0.2 \AA$ longer than the uncorrected value for the minimum geometry. Differences in the order of $1.2 \AA$ are obtained for the TS. The effect on the angular parameters is meaningful, mostly at the TS, where $\alpha$ and $\beta^{\prime}$ parameters change from $71.6^{\circ}$ and $71.7^{\circ}$ to $86.7^{\circ}$ and $79.5^{\circ}$, respectively. Neglect of the relaxation term does not change this situation. Relaxation energy terms are negligible. The largest distortions are found in the bond angles $a \mathrm{SOO}$ and $a \mathrm{HCH}$, belonging to the $\mathrm{SO}_{2}$ and $\mathrm{C}_{2} \mathrm{H}_{4}$ molecule, respectively. However, differences of 0.009 and $0.022 \AA$ in the intermolecular distance are obtained. Note that neglect of the relaxation term lengthens slightly the value of $R$ for the minimum, whereas the effect is opposite for the transition state. Table VI shows the values obtained for the rotational barrier; in this case, the relaxation term does not have a large effect. Small differences of $\approx 4-5$ $\mathrm{cm}^{-1}$ are observed. However, the CP-correction is mandatory; uncorrected values were three times larger than both the s.p.CP- and $\mathrm{CP}$-corrected. $\mathrm{CP}$-corrected values agree better with the estimated experimental value of $30 \mathrm{~cm}^{-1}$ proposed by Andrews et al. ${ }^{31}$

\section{CONCLUSIONS}

The counterpoise correction is found to be mandatory for these weakly bonded systems. The effects on the barrier to internal rotation energy and geometrical parameters have been analyzed. The assumptions made by Rayón et al. ${ }^{24}$ concerning the evaluation of energy barriers to rotation are incorrect from a conceptual point of view. The s.p.CP-

TABLE V. Geometrical parameters, electronic energies, and relaxation contribution for the transition state structure of the $\mathrm{C}_{2} \mathrm{H}_{4} \cdots \mathrm{SO}_{2}$ complex calculated at MP2, s.p.CP-MP2, and CP-MP2 levels of theory (see Fig. 2). Number of basis functions is shown. The second half collects the values obtained in the calculations where the intramolecular parameters where frozen to the values they have in the free monomers. Relaxation energy is calculated using Eq. (6).

\begin{tabular}{lcccccccc}
\hline \hline \multicolumn{1}{c}{ Method } & $\begin{array}{c}\text { Basis } \\
\text { functions }\end{array}$ & $\begin{array}{c}\text { Electronic energy } \\
\text { (hartrees) }\end{array}$ & $\begin{array}{c}R \\
(\AA)\end{array}$ & $\begin{array}{c}\alpha \\
(\mathrm{deg})\end{array}$ & $\begin{array}{c}\beta^{\prime} \\
(\mathrm{deg})\end{array}$ & $\begin{array}{c}a \mathrm{SOO} \\
(\mathrm{deg})\end{array}$ & $\begin{array}{c}a \mathrm{HCH} \\
(\mathrm{deg})\end{array}$ & $\begin{array}{c}\text { Relaxation } \\
\text { energy } \\
\left(\mathrm{cm}^{-1}\right)\end{array}$ \\
\hline $\begin{array}{l}\text { MP2/d95(d,p) } \\
\text { s.p.CP-corrected }\end{array}$ & 108 & -626.0434220 & 3.619 & 71.6 & 71.8 & 108.7 & 121.3 & 0.5 \\
CP-MP2/d95 $(d, p)$ & 108 & -626.0387983 & 3.732 & 86.7 & 79.5 & 108.7 & 121.3 & 0.5 \\
$\begin{array}{l}\text { MP2/d95 }(d, p) \\
\text { s.p.CP-corrected }\end{array}$ & 108 & -626.0430684 & 3.597 & 73.4 & 73.4 & $108.9^{\mathrm{a}}$ & $120.0^{\mathrm{a}}$ & $0^{\mathrm{b}}$ \\
CP-MP2/d95 $(d, p)$ & 108 & -626.0384377 & 3.726 & 87.1 & 80.3 & $108.9^{\mathrm{a}}$ & $120.0^{\mathrm{a}}$ & $0^{\mathrm{b}}$ \\
\hline \hline
\end{tabular}

aptimized parameters for $\mathrm{C}_{2} \mathrm{H}_{4}$ and $\mathrm{SO}_{2}$ isolated systems. 


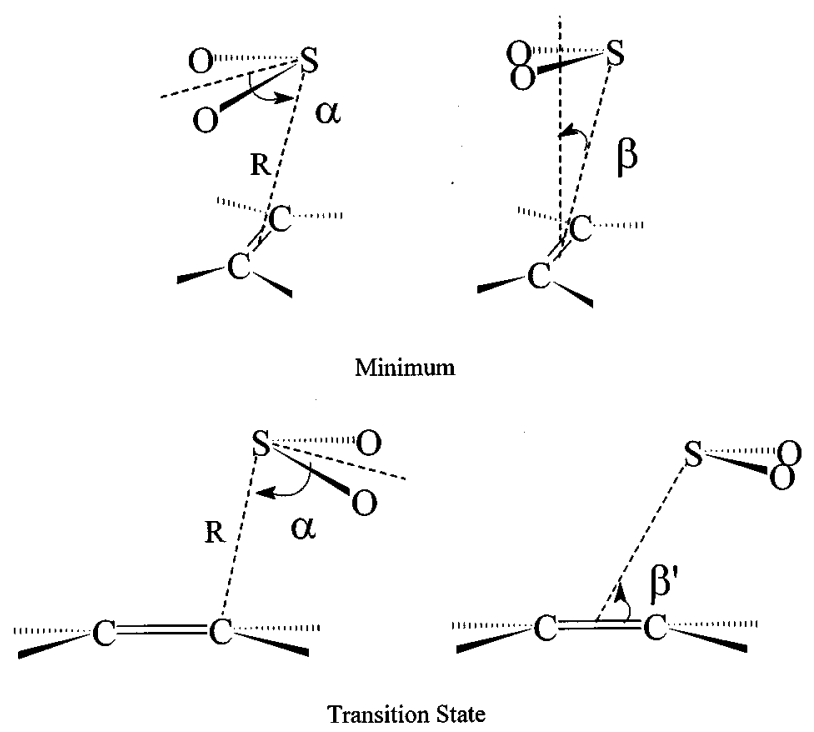

FIG. 2. Structures involved in the internal rotation of the $\mathrm{C}_{2} \mathrm{H}_{4} \cdots \mathrm{SO}_{2}$ system. Intermolecular parameters are indicated.

correction overestimates the BSSE. The CP-correction term is strongly geometry dependent; CP-corrected optimization must be carried out to obtain accurate BSSE-free geometry for further vibrational analysis.

Furthermore, the fragment relaxation energy cannot be seen as an additional term to the rotational barrier. The effect of the fragment relaxation on the intermolecular parameters probed to be very important for the $\mathrm{BF}_{3} \cdots \mathrm{NH}_{3}$ complex, the $\mathrm{CP}$-correction not changing this situation. The $\mathrm{CP}$-correction scheme can be successfully applied despite the relaxation contribution not being taken into account.

In terms of rotational barrier height, the single point $\mathrm{CP}-$ corrected and CP-corrected values are very similar. However, anharmonic effects can be very important in the hydrogen-bonded and van der Waals complexes..$^{22,32-34}$ For instance, for several water clusters, Jung et al. ${ }^{34}$ have shown that the anharmonic correction modifies by $100 \%$ the vibrational frequencies of intermolecular modes. Moreover, large

TABLE VI. Electronic energies $\left(\mathrm{cm}^{-1}\right)$ for the internal rotation barrier in the $\mathrm{C}_{2} \mathrm{H}_{4} \cdots \mathrm{SO}_{2}$ complex. In parentheses the values obtained are neglecting the relaxation term.

\begin{tabular}{lrr}
\hline \hline \multicolumn{1}{c}{ Method } & \multicolumn{2}{c}{ Rotational barrier $\left(\mathrm{cm}^{-1}\right)$} \\
\hline MP2/d95 $(d, p)$ & 166 & $(161)$ \\
s.p.CP-corrected & 46 & $(37)$ \\
CP-MP2/d95 $(d, p)$ & 47 & $(43)$ \\
\hline \hline
\end{tabular}

amplitude modes related to flat potential energy surfaces are poorly described using the rigid rotor-harmonic oscillator approach. ${ }^{35}$ This is the case of internal rotation motions. Thus, ZPVE corrections using CP-corrected harmonic and anharmonic frequencies for the intermolecular vibrational modes should be considered and their study is in progress in our laboratory.

\section{ACKNOWLEDGMENTS}

This work has been funded through the DGICYT Project No. PB95-0762. One of us (P.S.) acknowledges the financial support by CIRIT Grant No. 1998FI-00564.

${ }^{1}$ E. Clementi, J. Chem. Phys. 46, 3851 (1967).

${ }^{2}$ N. R. Kestner, J. Chem. Phys. 48, 252 (1968).

${ }^{3}$ S. Simon, M. Duran, and J. J. Dannenberg, J. Chem. Phys. 105, 11024 (1996).

${ }^{4}$ B. Paizs, P. Salvador, M. Duran, and S. Suhai, J. Comput. Chem. (to be published).

${ }^{5} \mathrm{P}$. Salvador and M. Duran (in preparation).

${ }^{6}$ P. Hobza and Z. Havlas, Theor. Chem. Acc. 99, 372 (1998).

${ }^{7}$ S. S. Xantheas, J. Chem. Phys. 104, 8821 (1996).

${ }^{8}$ K. A. Peterson and T. H. Dunning, Jr., J. Chem. Phys. 102, 2032 (1995).

${ }^{9}$ T. Van Mourik, A. K. Wilson, K. A. Peterson, D. E. Woon, and T. H. Dunning, Jr., Adv. Quantum Chem. 31, 105 (1998).

${ }^{10}$ A. J. Sadlej and P. Mazurek, J. Mol. Struct. 337, 129 (1995).

${ }^{11}$ I. Mayer, Int. J. Quantum Chem. 23, 341 (1983).

${ }^{12}$ I. Mayer, Á. Vivók, and P. Valiron, Chem. Phys. Lett. 224, 166 (1994).

${ }^{13}$ B. Paizs and S. Suhai, J. Comput. Chem. 18, 694 (1997).

${ }^{14}$ I. Mayer and Á. Vivók, Chem. Phys. Lett. 136, 115 (1987).

${ }^{15}$ I. Mayer and P. Valiron, J. Chem. Phys. 108, 3360 (1998).

${ }^{16}$ A. Famulari, M. Raimondi, M. Sironi, and E. Gianinetti, Chem. Phys. 232, 275 (1998).

${ }^{17}$ S. F. Boys and F. Bernardi, Mol. Phys. 19, 553 (1970).

${ }^{18}$ H. B. Jansen and P. Ros, Chem. Phys. Lett. 3, 140 (1969).

${ }^{19}$ M. Gutowski and G. Chalasinski, J. Chem. Phys. 98, 5540 (1993).

${ }^{20}$ M. Gutowski, J. G. C. M. Van Duijneveldt-van de Rijdt, J. H. Van Lenthe, and F. B. Van Duijneveldt, J. Chem. Phys. 98, 4728 (1993).

${ }^{21}$ D. B. Cook, J. A. Sordo, and T. Sordo, Int. J. Quantum Chem. 48, 385 (1993).

${ }^{22}$ K. Szawlewicz and B. Jeziorski, J. Chem. Phys. 109, 1198 (1998).

${ }^{23}$ G. Lendvay and I. Mayer, Chem. Phys. Lett. 297, 365 (1998).

${ }^{24}$ V. M. Rayón and J. A. Sordo, Theor. Chem. Acc. 99, 68 (1998).

${ }^{25}$ I. Mayer and P. R. Surján, Chem. Phys. Lett. 191, 497 (1992).

${ }^{26}$ M. J. Frisch, G. W. Trucks, H. B. Schlegel, P. M. W. Gill, B. G. Johnson, M. A. Robb, J. R. Cheeseman, T. A. Keith, G. A. Petersson, J. A. Montgomery, K. Raghavachari, M. A. Al-Laham, V. G. Zakrzewski, J. V. Ortiz, J. B. Foresman, J. Cioslowski, B. B. Stefanov, A. Nanayakkara, M. Challacombe, C. Y. Peng, P. Y. Ayala, W. Chen, M. W. Wong, J. L. Andres, E. S. Reploge, R. Gomperts, R. L. Martin, D. J. Fox, J. S. Binkley, D. J. Defrees, J. Baker, J. P. Stewart, M. Head-Gordon, C. Gonzalez, and J. A. Pople, Gaussian, Inc., Pittsburgh, Pennsylvania, 1995.

${ }^{27}$ C. Moller and M. S. Plesset, Phys. Rev. 46, 618 (1934).

${ }^{28}$ T. H. Dunning, Jr., J. Chem. Phys. 53, 2823 (1970).

${ }^{29}$ P. Pulay, J. Comput. Chem. 3, 556 (1982).

${ }^{30}$ R. Fletcher, Practical Methods of Optimization (Wiley, New York, 1980), Vol. I.

${ }^{31}$ A. M. Andrews, K. W. Hillig II, and R. L. Kuczkowski, J. Chem. Phys. 96, 1784 (1992).

${ }^{32}$ J. E. Del Benne and M. T. Jordan, J. Chem. Phys. 108, 3205 (1998).

${ }^{33}$ S. Scheiner, Annu. Rev. Phys. Chem. 45, 23 (1994).

${ }^{34}$ J. O. Jung and R. B. Gerber, J. Chem. Phys. 105, 10332 (1996).

${ }^{35}$ C. Muñoz-Caro and A. Niño, J. Phys. Chem. A 101, 4128 (1997). 